NASA/CR-1998-208672

$$
\begin{aligned}
& \text { IN-7/ ICOMP-98-07 } \\
& 4 / 32203
\end{aligned}
$$
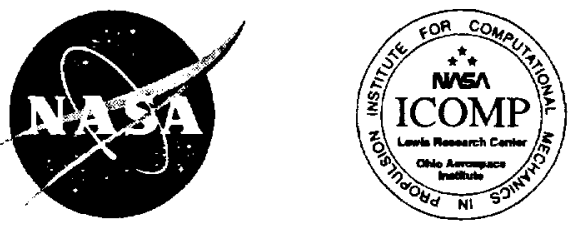

\title{
High-Accuracy Compact MacCormack-Type Schemes for Computational Aeroacoustics
}

R. Hixon

Institute for Computational Mechanics in Propulsion, Cleveland, Ohio

E. Turkel

Institute for Computational Mechanics in Propulsion, Cleveland, Ohio, and Tel Aviv University, Tel Aviv, Israel 
Since its founding, NASA has been dedicated to the advancement of aeronautics and space science. The NASA Scientific and Technical Information (STI) Program Office plays a key part in helping NASA maintain this important role.

The NASA STI Program Office is operated by Langley Research Center, the Lead Center for NASA's scientific and technical information. The NASA STI Program Office provides access to the NASA STI Database, the largest collection of aeronautical and space science STI in the world. The Program Office is also NASA's institutional mechanism for disseminating the results of its research and development activities. These results are published by NASA in the NASA STI Report Series, which includes the following report types:

- TECHNICAL PUBLICATION. Reports of completed research or a major significant phase of research that present the results of NASA programs and include extensive data or theoretical analysis. Includes compilations of significant scientific and technical data and information deemed to be of continuing reference value. NASA's counterpart of peerreviewed formal professional papers but has less stringent limitations on manuscript length and extent of graphic presentations.

- TECHNICAL MEMORANDUM. Scientific and technical findings that are preliminary or of specialized interest, e.g., quick release reports, working papers, and bibliographies that contain minimal annotation. Does not contain extensive analysis.

- CONTRACTOR REPORT. Scientific and technical findings by NASA-sponsored contractors and grantees.
- CONFERENCE PUBLICATION. Collected papers from scientific and technical conferences, symposia, seminars, or other meetings sponsored or cosponsored by NASA.

- SPECIAL PUBLICATION. Scientific, technical, or historical information from NASA programs, projects, and missions, often concerned with subjects having substantial public interest.

- TECHNICAL TRANSLATION. Englishlanguage translations of foreign scientific and technical material pertinent to NASA's mission.

Specialized services that complement the STI Program Office's diverse offerings include creating custom thesauri, building customized data bases, organizing and publishing research results ... . even providing videos.

For more information about the NASA STI Program Office, see the following:

- Access the NASA STI Program Home Page at http://www.sti.nasa.gov

- E-mail your question via the Internet to help@sti.nasa.gov

- Fax your question to the NASA Access Help Desk at (301) 621-0134

- Telephone the NASA Access Help Desk at (301) 621-0390

- Write to: NASA Access Help Desk NASA Center for AeroSpace Information 7121 Standard Drive Hanover, MD 21076 
NASA/CR-1998-208672
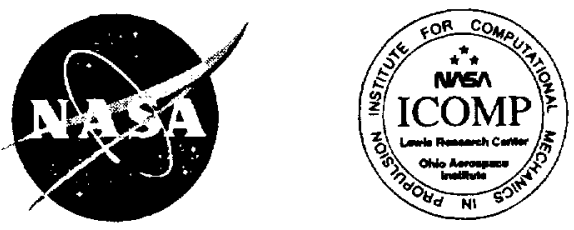

\section{High-Accuracy Compact MacCormack-Type Schemes for Computational Aeroacoustics}

\section{R. Hixon}

Institute for Computational Mechanics in Propulsion, Cleveland, Ohio

E. Turkel

Institute for Computational Mechanics in Propulsion, Cleveland, Ohio, and Tel Aviv University, Tel Aviv, Israel

Prepared under Cooperative Agreement NCC3-531

National Aeronautics and

Space Administration

Lewis Research Center 


\section{Acknowledgments}

This work was carried out under grant NCC3-531 from the NASA Lewis Research Center.

Dr. L.A. Povinelli was the Technical Monitor.

Available from

NASA Center for Aerospace Information

7121 Standard Drive

Hanover, MD 21076

Price Code: A03
National Technical Information Service 5285 Port Royal Road Springfield, VA 22100 


\title{
High-ACCURACY COMPACT MACCORMACK-TYPE SCHEMES FOR COMPUTATIONAL AEROACOUSTICS
}

\author{
R. Hixon \\ Institute for Computational Mechanics in Propulsion (ICOMP) \\ NASA Lewis Research Center, Cleveland, $\mathrm{OH} 44135$ \\ E. Turkel \\ Institute for Computational Mechanics in Propulsion (ICOMP) \\ NASA Lewis Research Center, Cleveland, OH 44135 \\ and \\ Tel Aviv University, Tel Aviv, Israel
}

\begin{abstract}
Using MacCormack-type methods, a new class of highly accurate compact MacCormack-type schemes is derived which does not require a tridiagonal matrix inversion to obtain the spatial derivatives. Two example schemes are shown, and results of these schemes for three linear and nonlinear CAA Benchmark Problems are presented.
\end{abstract}

\section{Introduction}

Computational aeroacoustics is concerned with the time-accurate solution of flow and acoustic phenomena over long periods of time. To accomplish this goal, high-order finite-difference schemes and optimized schemes have been developed [e.g., Refs. 1-6].

There are two main classes of high-accuracy finite-difference schemes: explicit schemes and compact schemes. Explicit schemes employ large computational stencils for accuracy, while compact schemes use smaller stencils by using the flux derivatives as independent variables at each grid point. While compact schemes are more accurate than the equivalent explicit scheme, solving for each flux derivative requires a scalar tridiagonal or pentadiagonal matrix inversion. 
Recently, a new class of high-accuracy explicit MacCormack-type schemes have been intro-

duced for computational aeroacoustics. ${ }^{6}$ Applying this methodology to compact schemes has resulted in a new class of compact MacCormack-type schemes which use three-point stencils while retaining high accuracy.

\section{Mathematical Formulation}

Consider the 1-D equation:

$$
\frac{\partial U}{\partial t}+\frac{\partial F}{\partial x}=0
$$

A general compact spatial derivative with a three-point stencil can be written to calculate the spatial derivative:

$$
\alpha D_{i-1}+(1-2 \alpha) D_{i}+\alpha D_{i+1}=\left(\frac{1}{\Delta x}\right)\left(\beta F_{i+1}-\beta F_{i-1}\right)
$$

where $D_{i}$ is the spatial derivative of $F$ at point $i$.

The standard Pade' compact scheme ${ }^{1}$ has the coefficients:

$$
\begin{aligned}
\alpha & =\frac{1}{6} \\
\beta & =\frac{1}{2}
\end{aligned}
$$


This gives fourth-order spatial accuracy. Since the value of the derivative at a point depends on the value of its nearest neighbors, a scalar tridiagonal matrix must be solved to obtain the spatial derivative.

Equation (2) can be written in matrix form as:

$$
[A]\{D\}=[B]\{F\}
$$

where $[A]$ and $[B]$ are tridiagonal matrices. To solve Eq. (4), the matrix [A] must be inverted:

$$
\{D\}=[A]^{-1}[B]\{F\}
$$

In a MacCormack-type scheme, the derivative operator is split into forward and backward operators such that:

$$
\{D\}=\frac{\left\{D^{F}\right\}+\left\{D^{B}\right\}}{2}
$$

The derivative operators can be written:

$$
\begin{aligned}
& \left\{D^{F}\right\}=\left[A^{F}\right]^{-1}\left[B^{F}\right]\{F\} \\
& \left\{D^{B}\right\}=\left[A^{B}\right]^{-1}\left[B^{B}\right]\{F\}
\end{aligned}
$$


Substituting into Eq. (6),

$$
\{D\}=\frac{1}{2}\left(\left[A^{F}\right]^{-1}\left[B^{F}\right]+\left[A^{B}\right]^{-1}\left[B^{B}\right]\right)\{F\}
$$

Multiplying through, we obtain:

$$
\left[A^{F}\right]\left[A^{B}\right]\{D\}=\frac{1}{2}\left(\left[A^{B}\right]\left[B^{F}\right]+\left[A^{F}\right]\left[B^{B}\right]\right) F
$$

Notice that Eq. (10) is only valid if:

$$
\left[A^{F}\right]\left[A^{B}\right]=\left[A^{B}\right]\left[A^{F}\right]
$$

Equation (11) is true for all tridiagonal matrices with constant diagonals.

To avoid a tridiagonal matrix inversion, we use bidiagonal matrices for the forward and backward operators. Writing out explicitly, we have:

$$
\begin{aligned}
& a^{B} D^{B}{ }_{i-1}+\left(1-a^{B}\right) D^{B}{ }_{i}= \\
& \left(\frac{1}{\Delta x}\right)\left(k^{B} F_{i-1}-\left(k^{B}+m^{B}\right) F_{i}+m^{B} F_{i+1}\right) \\
& \left(1-c^{F}\right) D^{F}{ }_{i}+c^{F} D^{F}{ }_{i+1}= \\
& \left(\frac{1}{\Delta x}\right)\left(k^{F} F_{i-1}-\left(k^{F}+m^{F}\right) F_{i}+m^{F} F_{i+1}\right)
\end{aligned}
$$


Using this, we find that at point i:

$$
\begin{aligned}
& {\left.\left[A^{F}\right]\left[A^{B}\right]\{D\}\right|_{i}=} \\
& a^{B}\left(1-c^{F}\right) D_{i-1}+\left(1-a^{B}\right)\left(1-c^{F}\right) D_{i}+c^{F}\left(1-a^{B}\right) D_{i+1}
\end{aligned}
$$

Matching the coefficients in Eq. (14) with those on the left side of Eq. (2) gives:

$$
\begin{gathered}
c^{F}=a^{B} \\
a^{B}=\frac{1 \pm \sqrt{1-4 \alpha}}{2}=\frac{1 \pm \sqrt{\frac{1}{3}}}{2}
\end{gathered}
$$

On the explicit side, we find:

$$
\begin{aligned}
& \left.\frac{1}{2}\left(\left[A^{B}\right]\left[B^{F}\right]+\left[A^{F}\right]\left[B^{B}\right]\right)\{F\}\right|_{i}= \\
& \frac{1}{2}\left(\left(a^{B} k^{F}\right) F_{i-2}\right. \\
& +\left(\left(1-c^{F}\right) k^{B}+\left(1-a^{B}\right) k^{F}-a^{B}\left(k^{F}+m^{F}\right)\right) F_{i-1}+ \\
& \left(c^{F} m^{B}-\left(1-c^{F}\right)\left(k^{B}+m^{B}\right)-\left(1-a^{B}\right)\left(k^{F}+m^{F}\right)+a^{B} m^{F}\right) F_{i} \\
& +\left(\left(1-c^{F}\right) m^{B}-c^{F}\left(k^{B}+m^{B}\right)+\left(1-a^{B}\right) k^{F}\right) F_{i+1} \\
& \left.+\left(c^{F} m^{B}\right) F_{i+2}\right)
\end{aligned}
$$

Matching the coefficients in Eq. (16) with Eq. (2) gives: 


$$
\begin{gathered}
k^{F}=-m^{B}=0 \\
k^{B}=-m^{F}=-2 \beta=-1
\end{gathered}
$$

Performing a Taylor series expansion of the forward and backward operators gives:

$$
\begin{aligned}
& D^{F}=F_{x}+\Delta x \frac{\sqrt{3}}{6} F_{x x}-(\Delta x)^{3} \frac{\sqrt{3}}{72} F_{x x x x}+O(\Delta x)^{4} \\
& D^{B}=F_{x}-\Delta x \frac{\sqrt{3}}{6} F_{x x}+(\Delta x)^{3} \frac{\sqrt{3}}{72} F_{x x x x}+O(\Delta x)^{4}
\end{aligned}
$$

Clearly, a fourth-order accurate central difference is recovered when the forward and backward operators are substituted into Eq. (6). Using the terminology of Ref. [6], this method is a 4/2 scheme. This terminology refers to the order of the underlying fourth-order central difference and the leading error term in the first-order biased differences.

Notice that, since bidiagonal matrices are used to calculate the derivatives, the local value of $D$ may be found using the value of $\mathrm{D}$ on one side only. By sweeping in the proper direction, the values of D may be found explicitly.

By using an additional explicit point and following the method used in Ref. [6], the order of the forward and backward operators may be increased to third order, resulting in a $4 / 4$ scheme. This scheme is defined as:

$$
c^{F}=a^{B}=\frac{1}{3}
$$




$$
\begin{aligned}
& k^{F}=-m^{B}=-\frac{1}{6} \\
& k^{B}=-m^{F}=-\frac{5}{6}
\end{aligned}
$$

Consequently, one extra point is used in the explicit operator to achieve the increase in order. The Taylor series expansion of the forward and backward operators is:

$$
\begin{aligned}
& D^{F}=F_{x}-(\Delta x)^{3} \frac{1}{36} F_{x x x x}+O(\Delta x)^{4} \\
& D^{B}=F_{x}+(\Delta x)^{3} \frac{1}{36} F_{x x x x}+O(\Delta x)^{4}
\end{aligned}
$$

Substituting the coefficients of Eq. (19) into Eq. (16) reveals that the $4 / 4$ scheme reduces to the following fourth-order compact scheme:

$$
\begin{aligned}
& \frac{2}{9} D_{i-1}+\frac{5}{9} D_{i}+\frac{2}{9} D_{i+1}= \\
& \left(\frac{1}{\Delta x}\right)\left(\frac{1}{36} F_{i+2}+\frac{4}{9} F_{i+1}-\frac{4}{9} F_{i-1}-\frac{1}{36} F_{i-2}\right)
\end{aligned}
$$

This illustrates one very important difference between the explicit MacCormack-type schemes given in Ref. [6] and the compact MacCormack-type schemes described here. For explicit schemes, the order of the forward and backward operators may be changed without changing the underlying central difference (i.e., the sum of the forward and backward operators for the explicit $4 / 2$ and $4 / 4$ schemes is the same fourth-order central difference, giving identical dispersion per- 
formance), while the compact $4 / 2$ and $4 / 4$ schemes have completely different dispersion performance.

Notice that using a larger stencil on the forward and backward operators results in a larger equivalent stencil for the underlying difference. As shown in Ref. [8], this method can be used to derive a class of prefactored small-stencil high-order compact schemes.

\section{Time Marching Methods}

The original MacCormack scheme used a two-stage explicit time marching method:

$$
\begin{gathered}
U^{(1)}=U^{n}-\Delta t \delta^{F}\left[F\left(U^{n}\right)\right] \\
U^{n+1}=\frac{1}{2}\left(U^{n}+U^{(1)}-\Delta t \delta^{B}\left[F\left(U^{(1)}\right)\right]\right)
\end{gathered}
$$

This scheme is second-order accurate in time, and is fourth-order accurate in space using the $4 / 2$ compact-implicit scheme given in Eq. (15) for linear problems. To obtain fourth-order accuracy in space for nonlinear problems, one needs to permute the forward and backward differences (see Ref. [5]).

In addition, three multistage Runge-Kutta-type time marching methods for MacCormack-type schemes will be described. These methods were derived for MacCormack-type schemes in [6], and will be briefly described here. Using Eq. (1), a general six-stage Runge-Kutta MacCormacktype scheme can be defined as

$$
h^{(1)}=-\Delta t \delta^{F}\left[F\left(U^{n}\right)\right]
$$




$$
\begin{aligned}
& h^{(2)}=-\Delta t \delta^{B}\left[F\left(U^{n}+\alpha_{2} h^{(1)}\right)\right] \\
& h^{(3)}=-\Delta t \delta^{F}\left[F\left(U^{n}+\alpha_{3} h^{(2)}\right)\right] \\
& h^{(4)}=-\Delta t \delta^{B}\left[F\left(U^{n}+\alpha_{4} h^{(3)}\right)\right] \\
& h^{(5)}=-\Delta t \delta^{F}\left[F\left(U^{n}+\alpha_{5} h^{(4)}\right)\right]
\end{aligned}
$$

Table 1: Time Marching Schemes

\begin{tabular}{|l|l|l|l|l|}
\hline & RK2 & RK4 & $\begin{array}{c}\text { LDDRK 4- } \\
\text { 6 Step 1 }\end{array}$ & $\begin{array}{c}\text { LDDRK 4- } \\
\text { 6 Step 2 }\end{array}$ \\
\hline \hline$\alpha_{2}$ & 1 & $1 / 2$ & $1 / 2$ & 0.353323 \\
\hline$\alpha_{3}$ & 0 & $1 / 2$ & $1 / 2$ & 0.999597 \\
\hline$\alpha_{4}$ & 0 & 1 & 1 & 0.152188 \\
\hline$\alpha_{5}$ & 0 & 0 & 0 & 0.534216 \\
\hline$\alpha_{6}$ & 0 & 0 & 0 & 0.603907 \\
\hline$\beta_{1}$ & $1 / 2$ & $1 / 6$ & $1 / 6$ & 0.0467621 \\
\hline$\beta_{2}$ & $1 / 2$ & $1 / 3$ & $1 / 3$ & 0.137286 \\
\hline$\beta_{3}$ & 0 & $1 / 3$ & $1 / 3$ & 0.170975 \\
\hline$\beta_{4}$ & 0 & $1 / 6$ & $1 / 6$ & 0.197572 \\
\hline$\beta_{5}$ & 0 & 0 & 0 & 0.282263 \\
\hline$\beta_{6}$ & 0 & 0 & 0 & 0.165142 \\
\hline$c_{1}$ & 1 & 1 & 1 & 1 \\
\hline$c_{2}$ & $1 / 2$ & $1 / 2$ & $1 / 2$ & $1 / 2$ \\
\hline$c_{3}$ & 0 & $1 / 6$ & $1 / 6$ & $1 / 6$ \\
\hline$c_{4}$ & 0 & $1 / 24$ & $1 / 24$ & $1 / 24$ \\
\hline$c_{5}$ & 0 & 0 & 0 & 0.0162098 \\
\hline$c_{6}$ & 0 & 0 & 0 & 0.00286365 \\
\hline
\end{tabular}




$$
\begin{gathered}
h^{(6)}=-\Delta t \delta^{B}\left[F\left(U^{n}+\alpha_{6} h^{(5)}\right)\right] \\
U^{n+1}=U^{n}+\beta_{1} h^{(1)}+\beta_{2} h^{(2)}+\beta_{3} h^{(3)} \\
+\beta_{4} h^{(4)}+\beta_{5} h^{(5)}+\beta_{6} h^{(6)}
\end{gathered}
$$

where $\delta^{F}$ refers to a forward spatial difference and $\delta^{B}$ to a backward spatial difference. Normally, the order of forward and backward differences are interchanged every step to avoid numerical biasing.

The coefficients for the three time marching schemes are given in Table 1. The RK2 scheme is second-order accurate, while the RK4 and Hu, et. al's 4-6 LDDRK methods ${ }^{9}$ are both fourthorder accurate in a linear sense. However, for nonlinear problems, the RK4 scheme is fourthorder accurate while the 4-6 LDDRK method presented here reduces to third order accuracy. Notice that the 4-6 LDDRK method uses a two-step marching cycle; one step has four stages and the second step has six.

\section{Accuracy Analysis}

Consider a general MacCormack-type method, which is $k$ th order accurate in time. The underlying central difference is $l$ th order accurate in space, while the biased differences are $m$ th order accurate in space. An accuracy analysis using Eq. (1) will give:

$$
U(t+\Delta t)=U(t)+\frac{\partial U}{\partial t}+\vartheta\left(\Delta t^{k}, \Delta t \Delta x^{m+1}, \Delta x^{l}\right)
$$

From Eq. (18), the $4 / 2$ scheme will have a third-order time-space error term, while the $4 / 4$ 
scheme is a true fourth-order method.

A wavenumber analysis of these schemes gives a more complete picture of their linear performance and stability. The numerical wavenumber for a general three-point compact derivative (Eq. (2)) is defined as:

$$
\bar{\theta} \Delta x=\frac{-i\left(k e^{-i \theta \Delta x}-(k+m)+m e^{i \theta \Delta x}\right)}{\left(a e^{-i \theta \Delta x}+1-(a+c)+c e^{i \theta \Delta x}\right)}
$$

where

$$
-\pi \leq \theta \Delta x \leq \pi
$$

For the standard compact scheme, the numerical wavenumber is given by:

$$
\bar{\theta} \Delta x=\frac{\sin (\theta \Delta x)}{\left(\frac{2}{3}+\frac{1}{3} \cos (\theta \Delta x)\right)}
$$

Notice that the right side of Eq. (27) is purely real. This is a property of central-difference stencils.

For the 4/2 MacCormack-type scheme, the numerical wavenumber for the forward biased difference is: 


$$
\bar{\theta}^{F} \Delta x=\left(\frac{\sin (\theta \Delta x)}{\left(\frac{2}{3}+\frac{1}{3} \cos (\theta \Delta x)\right)}-\frac{\frac{i}{\sqrt{3}}(-1+\cos (\theta \Delta x))}{\left(\frac{2}{3}+\frac{1}{3} \cos (\theta \Delta x)\right)}\right)
$$

Similarly, the numerical wavenumber for the backward biased difference is:

$$
\bar{\theta}^{B} \Delta x=\left(\frac{\sin (\theta \Delta x)}{\left(\frac{2}{3}+\frac{1}{3} \cos (\theta \Delta x)\right)}+\frac{\frac{i}{\sqrt{3}}(-1+\cos (\theta \Delta x))}{\left(\frac{2}{3}+\frac{1}{3} \cos (\theta \Delta x)\right)}\right)
$$

There are two interesting things to observe in Eqs. (28) and (29). First, the real (dispersive) part of both equations are equal and identical to the real part of Eq. (27). Second, the imaginary

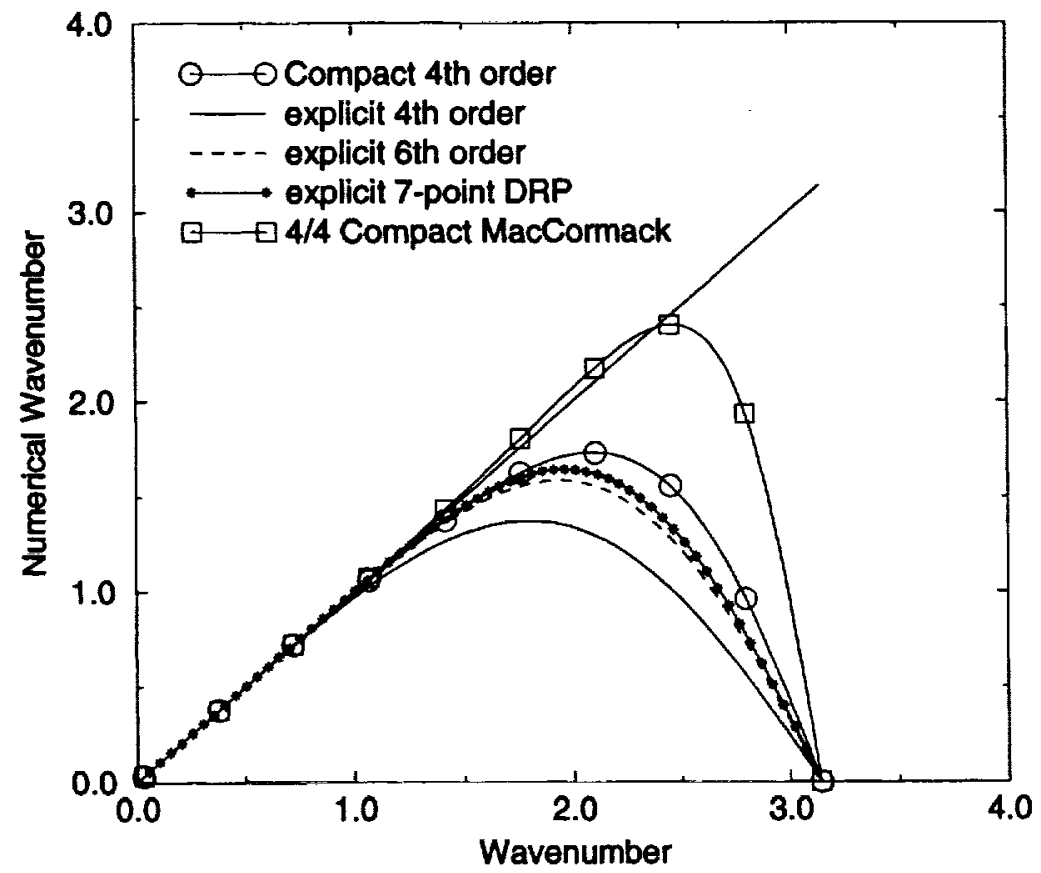

Figure 1: Dispersion Characteristics of Compact MacCormack-type Schemes 
(dissipative) parts are equal and opposite. This is a property of MacCormack-type schemes.

Likewise, the numerical wavenumbers for the $4 / 4$ scheme are:

$$
\begin{gathered}
\bar{\theta}^{F} \Delta x=\left(\frac{\left(\frac{2}{9} \sin (\theta \Delta x)-\frac{1}{18} \sin (2 \theta \Delta x)\right)}{\left(\frac{5}{9}+\frac{4}{9} \cos (\theta \Delta x)\right)}\right. \\
\left.-\frac{i\left(-\frac{5}{18}+\frac{2}{9} \cos (\theta \Delta x)+\frac{1}{18} \cos (2 \theta \Delta x)\right)}{\left(\frac{5}{9}+\frac{4}{9} \cos (\theta \Delta x)\right)}\right)
\end{gathered}
$$

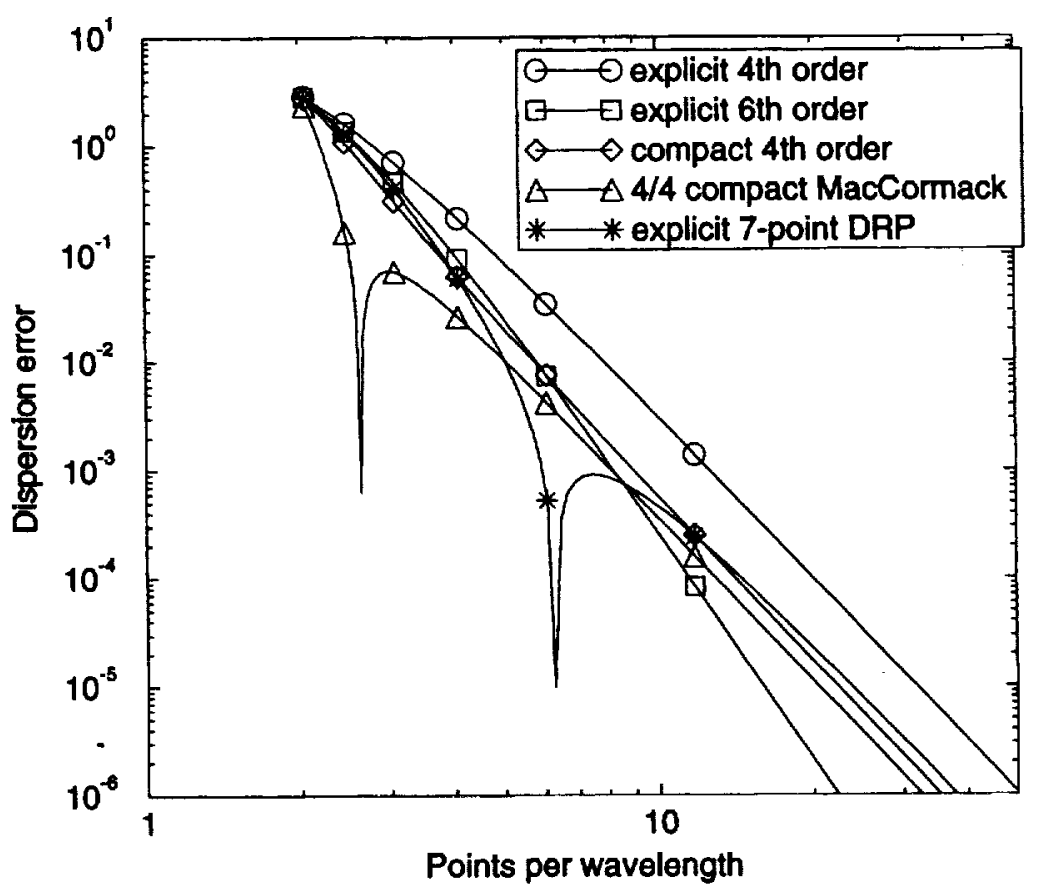

Figure 2: Dispersion Error Magnitude Comparison 


$$
\begin{gathered}
\bar{\theta}^{B} \Delta x=\left(\frac{\left(\frac{2}{9} \sin (\theta \Delta x)-\frac{1}{18} \sin (2 \theta \Delta x)\right)}{\left(\frac{5}{9}+\frac{4}{9} \cos (\theta \Delta x)\right)}\right. \\
\left.+\frac{i\left(-\frac{5}{18}+\frac{2}{9} \cos (\theta \Delta x)+\frac{1}{18} \cos (2 \theta \Delta x)\right)}{\left(\frac{5}{9}+\frac{4}{9} \cos (\theta \Delta x)\right)}\right)
\end{gathered}
$$

Figures 1 and 2 show the dispersion performance of these schemes, and compares them to explicit schemes such as 4th and 6th order central differences and Tam and Webb's 7-point DRP scheme. $^{3}$ Notice the improvement in the dispersion performance throughout the wavenumber range from the 4/4 scheme; unlike all explicit MacCormack-type schemes, it outperforms the scheme that it is based on.

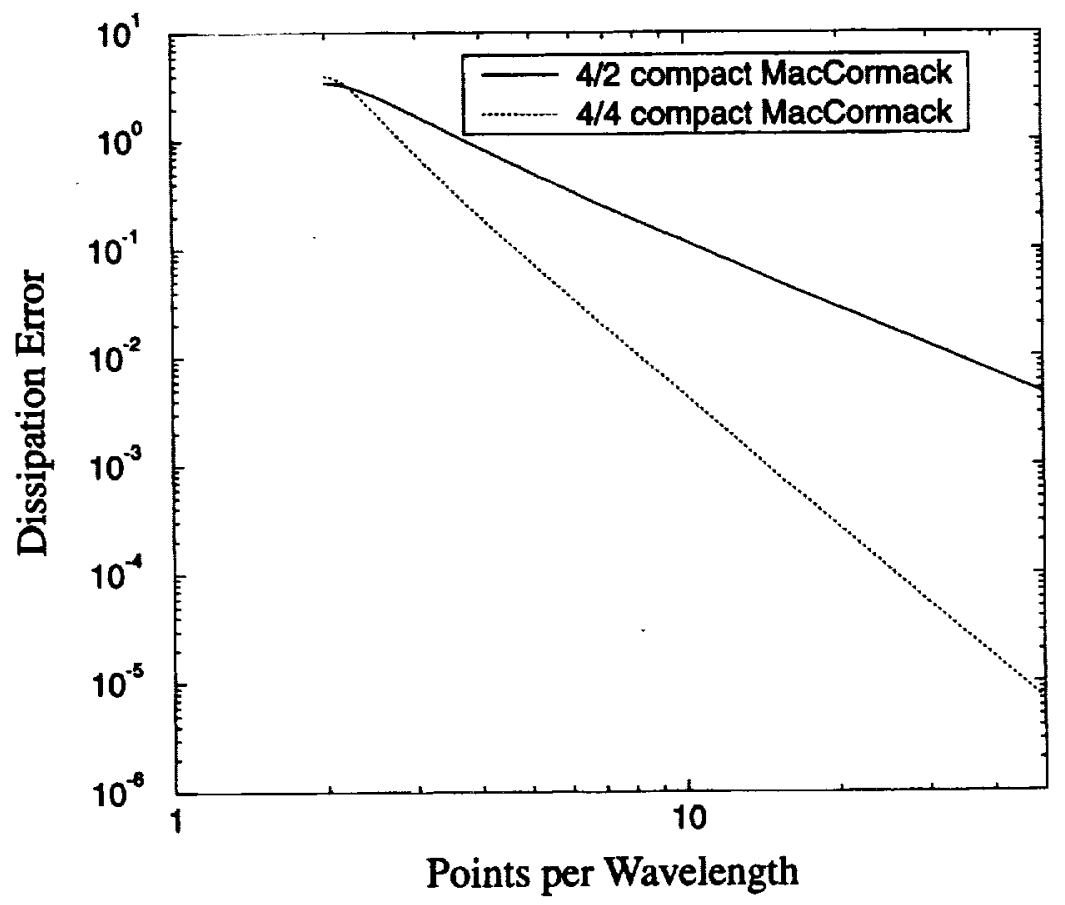

Figure 3: Dissipation Magnitude for Compact MacCormack-type Schemes 
Figure 3 shows the dissipation performance of the two MacCormack-type schemes. Again, the reduction in dissipation in the resolved wavenumber range from the $4 / 4$ scheme is evident.

\section{Performance and Stability of Compact MacCormack-type Schemes}

The numerical wavenumber of the forward and backward operators of a MacCormack-type scheme may be written as:

$$
\begin{aligned}
& \bar{\theta}^{F}=\bar{\theta}^{C}-i \delta \\
& \bar{\theta}^{B}=\bar{\theta}^{C}+i \delta
\end{aligned}
$$

Notice that the dispersion relation $\overline{\theta^{C}}$ is the same for both the forward and backward differences; however, the dissipative term $\delta$ is equal and opposite.

Putting these definitions into the time-marching schemes described above, we obtain:

$$
\begin{aligned}
U(t+\Delta t)= & \left(1+c_{1}(-i \Delta t) \bar{\theta}^{C}+\right. \\
& c_{2}(-i \Delta t)^{2}\left(\left(\bar{\theta}^{C}\right)^{2}+\delta^{2}\right)+ \\
& c_{3}(-i \Delta t)^{3} \bar{\theta}^{C}\left(\left(\bar{\theta}^{C}\right)^{2}+\delta^{2}\right)+ \\
& c_{4}(-i \Delta t)^{4}\left(\left(\bar{\theta}^{C}\right)^{2}+\delta^{2}\right)^{2}+ \\
& c_{5}(-i \Delta t)^{5} \bar{\theta}^{C}\left(\left(\bar{\theta}^{C}\right)^{2}+\delta^{2}\right)^{2}+ \\
& \left.c_{6}(-i \Delta t)^{6}\left(\left(\bar{\theta}^{C}\right)^{2}+\delta^{2}\right)^{3}\right) U(t) \\
& =\langle G(\theta)\rangle U(t)
\end{aligned}
$$

Using this equation, we can find the frequency response of the time-marching scheme, as well as its stability. For this stability analysis, the magnitude of $G(\theta)$ must be equal to or less than one for $0<\theta<\pi$. In this work, $\theta>0$ and $\theta=\pi$, which measure the long and short wavelength stabil- 
ity, are investigated analytically. As a matter of observation, if the scheme is stable for small $\theta$ and for $\theta=\pi$, it is almost always stable for all $\theta$. However, there is no mathematical proof that this has to be true.

Table 2 shows the results for the stability analysis, both analytical and numerical. Interestingly enough, the results for several of the schemes show that the most unstable wavenumber is not at $\theta->0$ or $\theta=\pi$. In particular, the LDDRK 4-6 scheme had its most unstable wavenumber at approximately $\theta=0.91 \pi$.

\section{Boundary Stencils for Compact MacCormack-type Schemes}

\section{1) Effect of Boundary Stencils on Interior Scheme Performance}

The numerical performance of the boundary stencil for a compact scheme has a much larger effect on the stability and accuracy of the scheme than the boundary stencil for the equivalent

Table 2: Stability Limits

\begin{tabular}{|l|l|l|l|l|}
\hline \multicolumn{1}{|c|}{ Scheme } & \multicolumn{1}{|c|}{$\begin{array}{c}\text { Time } \\
\text { marching }\end{array}$} & $\begin{array}{c}\text { CFL limit } \\
(\theta \rightarrow 0)\end{array}$ & $\begin{array}{c}\text { CFL limit } \\
(\theta \rightarrow \pi)\end{array}$ & $\begin{array}{c}\text { CFL limit } \\
\text { (numerical) }\end{array}$ \\
\hline \hline $4 / 2$ & RK2 & $\frac{1}{\sqrt{3}}$ & $\frac{1}{\sqrt{3}}$ & 0.577 \\
\hline & RK4 & (stable) & 1 & 1.0 \\
\hline & $\begin{array}{l}\text { LDDRK } \\
4-6\end{array}$ & (stable) & .934684 & 0.891 \\
\hline $4 / 4$ & RK2 & (unstable) & $\frac{1}{2}$ & (unstable) \\
\hline & RK4 & (stable) & $\frac{\sqrt{3}}{2}$ & 0.851 \\
\hline & $\begin{array}{l}\text { LDDRK } \\
4-6\end{array}$ & (stable) & .80946 & 0.747 \\
\hline
\end{tabular}


compact scheme. ${ }^{10,11}$ The reason for this is that the error from the boundary stencil can propagate many points into the computational domain.

Taking the $4 / 2$ scheme as an example, let us assume that we have an error $\varepsilon_{0}$ from the boundary at the beginning of the backward sweep. Defining $\varepsilon_{0}$ as:

$$
\varepsilon_{0}=\left(D^{B}\right)_{\text {interior }}-\left(D^{B}\right)_{\text {boundary }}
$$

where the subscript 'interior' refers to the spatial derivative that the interior scheme would have obtained and the subscript 'boundary' refers to the derivative obtained by the boundary stencil. It can be seen that we are defining the error with respect to the spatial derivative that the interior scheme would have obtained rather than the exact analytical derivative.

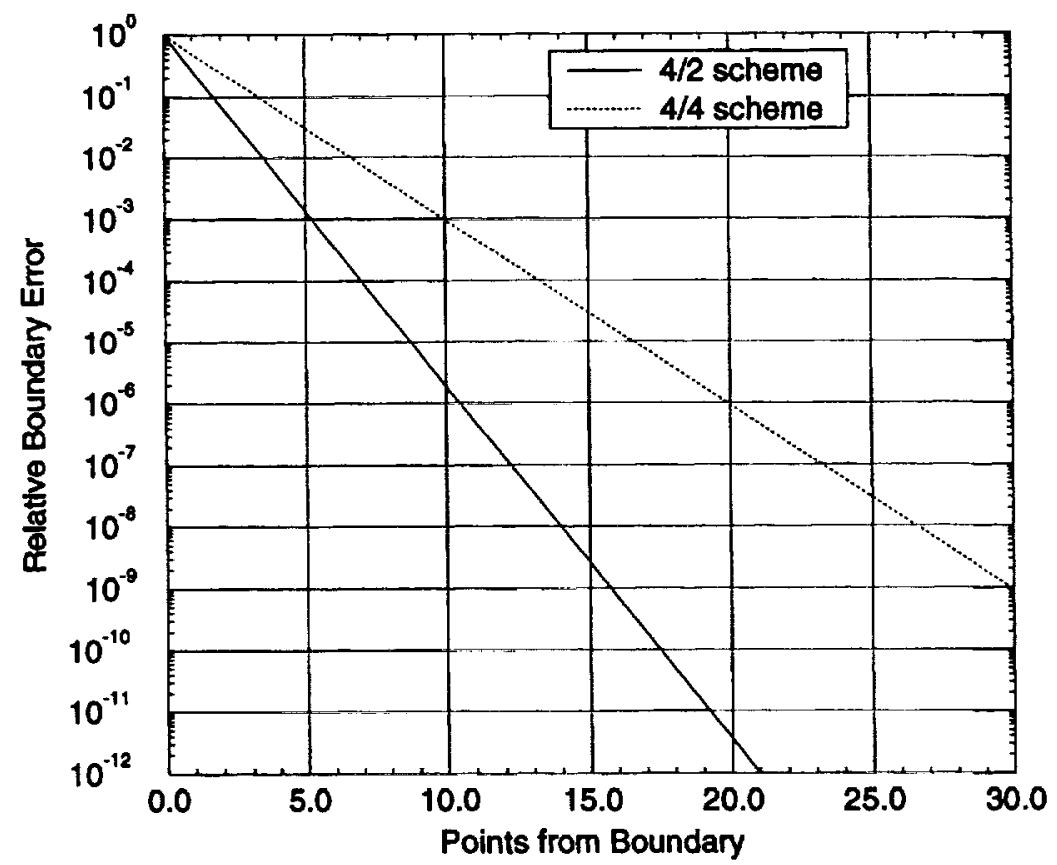

Figure 4: Effect of Boundary Stencil Error on Interior Solution 
Substituting into Eq. (12), we find that the error propagates inward from the boundary. The error for a derivative $\mathrm{i}$ grid points away from the boundary is:

$$
\left.\left(D^{B}\right)_{\text {interior }}\right|_{i}=\left.\left(D^{B}\right)_{b o u n d a r y}\right|_{i}+\left(-\frac{a^{B}}{1-a^{B}}\right)^{i} \varepsilon_{0}
$$

or

$$
\varepsilon_{i}=\left(-\frac{a^{B}}{1-a^{B}}\right)^{i} \varepsilon_{0}
$$

One point of interest is that the error due to the boundary stencil used at the start of the sweep has a much greater effect on the computed derivative than that of the boundary stencil used at the end of the sweep. Figure 4 illustrates the effect of boundary stencil error on the interior derivatives for the $4 / 2$ and $4 / 4$ schemes. It should be noted that the $4 / 2$ scheme is less affected by boundary stencil error than the $4 / 4$ scheme.

\section{2) Boundary Stencil Description}

One-sided, five-point, explicit boundary stencils were used for the two schemes described. These stencils were obtained by matching the Taylor series terms to the fourth order for the forward and backward stencils of each scheme. For space reasons, the stencils are not given here; however, the derivation is straightforward.

This is not the only option for boundary stencil specification. Another possibility is to define 
ghost points outside the computational domain and extrapolate data to these artificial points using fourth-order accurate extrapolation. In this way, the interior scheme can be used on the boundaries. For the fourth-order accurate compact schemes given here, only one ghost point is needed.

\section{3) Effect of Multistage Time Stepping on Accuracy at Boundaries}

The boundary condition specification for MacCormack-type schemes is not straightforward, due to the dissipative error terms in the one-sided differences. For example, the standard symmetry plane condition is set by using the method of images to set ghost values outside the domain. This, however, is only true for the first stage of a MacCormack-type method. For the second stage, the method of images is not correct; instead, the ghost values must be set in such a way that the end result is symmetric. For a two-stage RK2 method, this may be possible; for a four- or six-stage method, the errors can mount quickly. As noted above, the errors at the boundary will instantaneously propagate far into the domain at each stage. Thus, correct boundary condition specification is critical to the success and stability of compact MacCormacktype schemes.

\section{Benchmark Test Problems and Results}

Three test problems were chosen to investigate the numerical performance of the two compact MacCormack-type schemes. These problems are from the First and Second CAA Workshops. 12,13

\section{1) 1-D Linear Wave Propagation}

The first problem tested is the first linear problem given in the first CAA Workshop. The problem asks for the solution of the $1-\mathrm{D}$ linear convection equation at time $=400$ : 


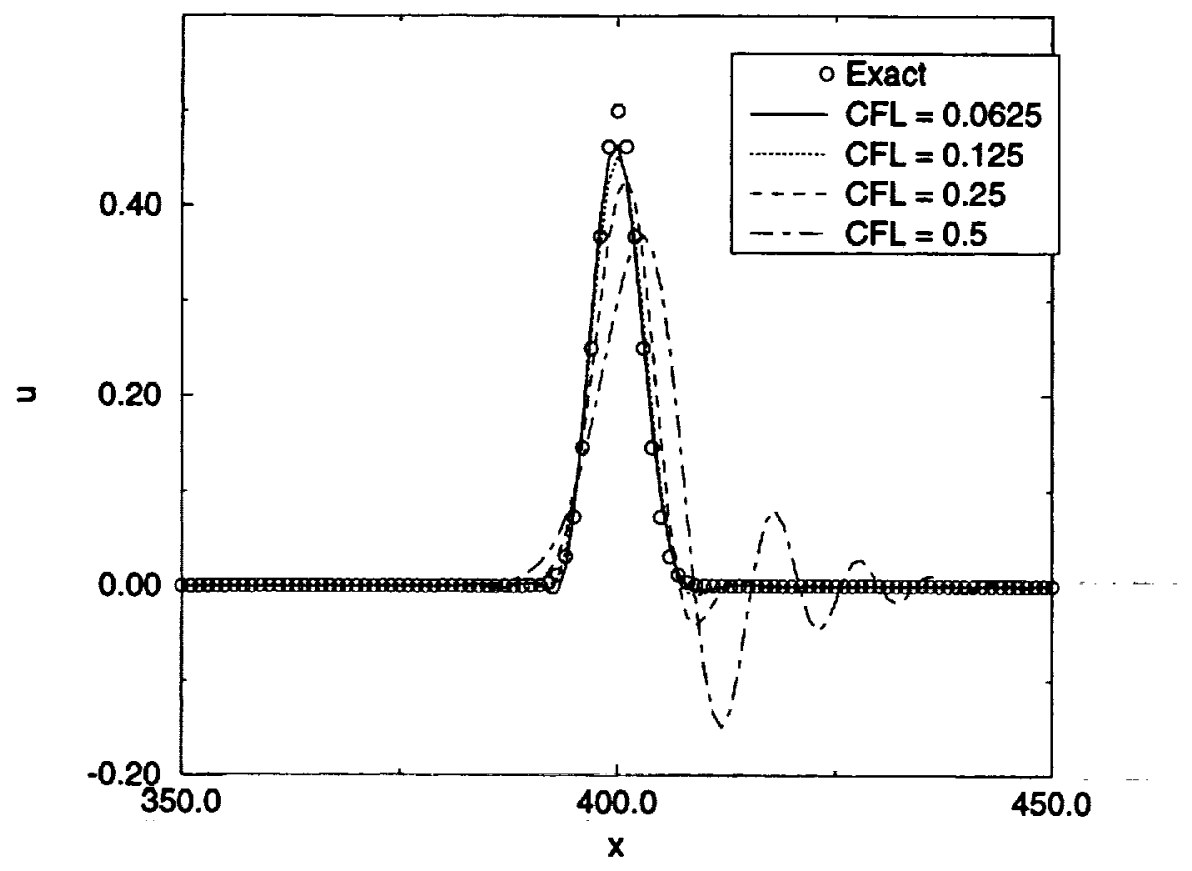

Figure 5: Performance of 2-4/2 Scheme on Benchmark Problem 1

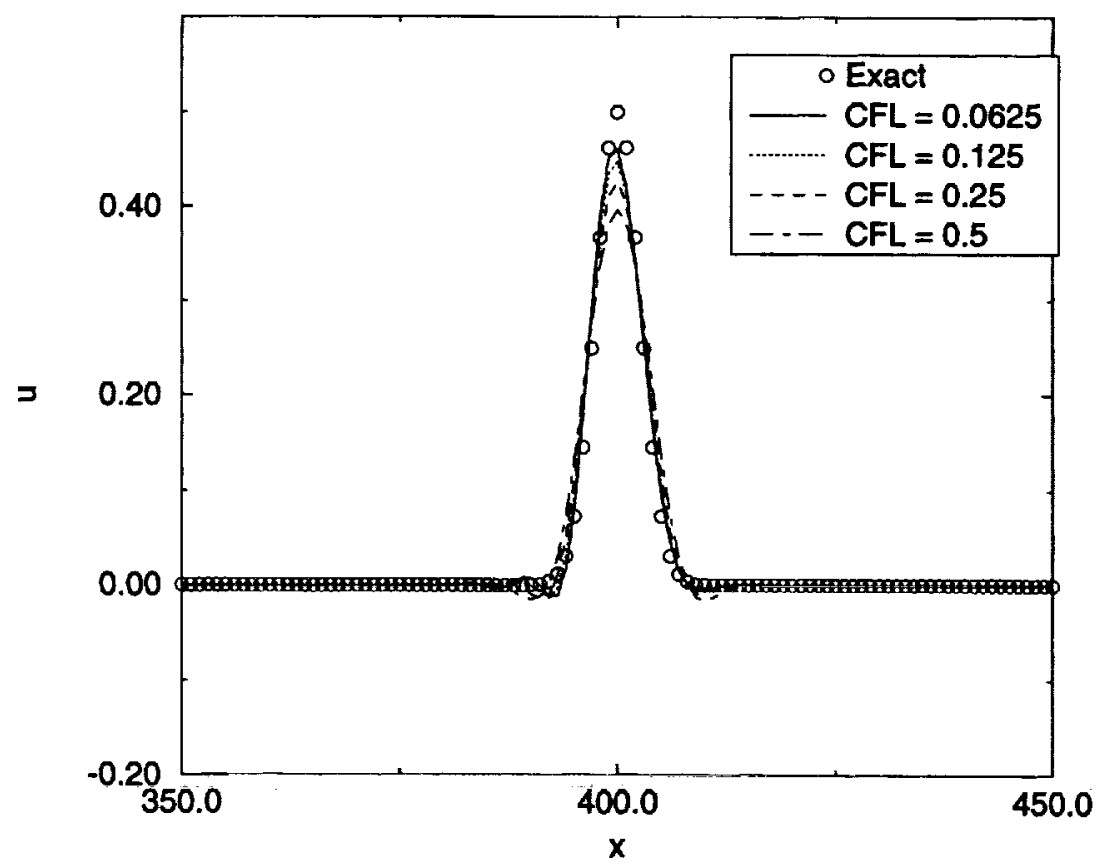

Figure 6: Performance of 4-4/2 Scheme on Benchmark Problem 1 


$$
\begin{aligned}
& U_{t}+U_{x}=0 \\
& U(x, 0)=\frac{1}{2} \exp \left(-\ln (2)\left(\frac{x}{3}\right)^{2}\right)
\end{aligned}
$$

where:

$$
\begin{aligned}
& \Delta x=1.0 \\
& -20 \leq x \leq 450
\end{aligned}
$$

Boundary condition specification is straightforward in this problem. At $x=-20$, which is the inflow boundary, the derivative of $U$ is set to zero. At $x=450$, which is the outflow boundary, the derivative of $\mathrm{U}$ is calculated explicitly from the interior.

Figures 5-7 show the results for the $4 / 2$ scheme used with each time-stepping method at varying time steps. As noted in Ref. [6], the RK2 time-stepping method shows a tendency to affect the

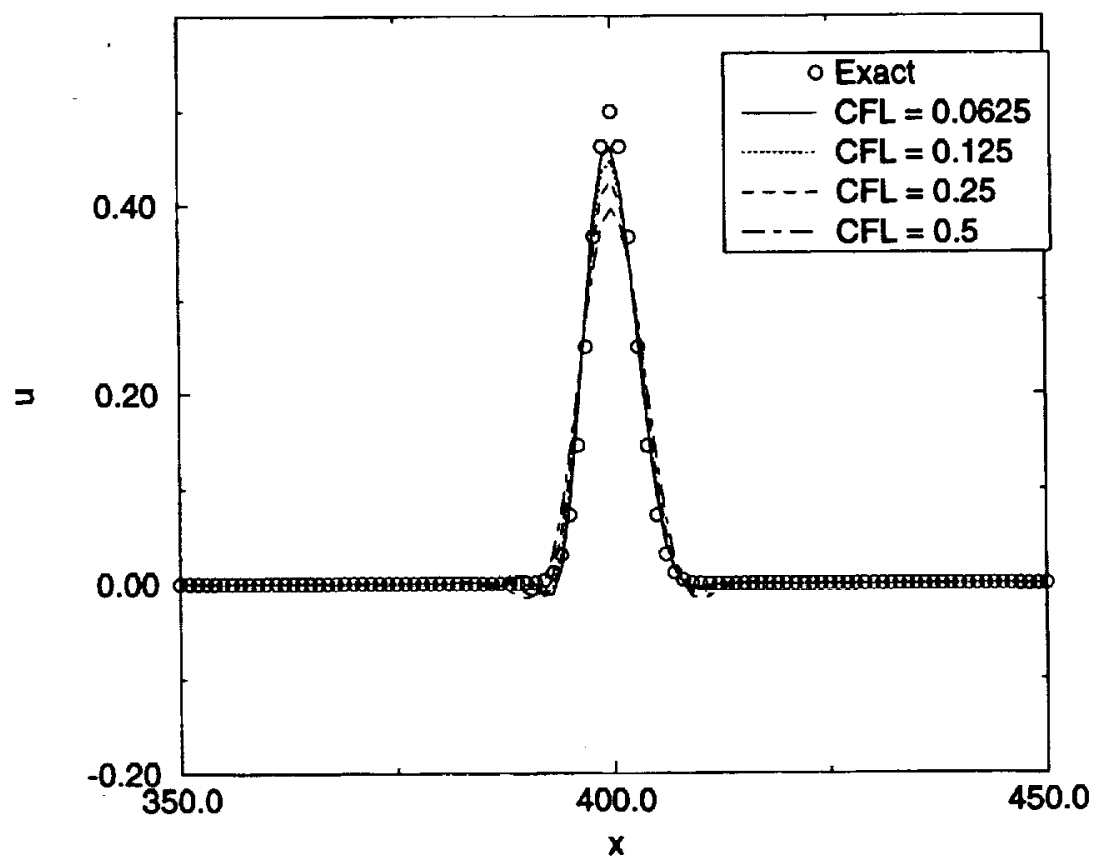

Figure 7: Performance of (4-6)-4/2 Scheme on Benchmark Problem 1 
dispersion as the time step increases, while the RK4 method tends to be dissipative at higher time steps. The LDDRK 4-6 method also dissipates at higher time steps when combined with the 4/2 scheme; however, this is more due to the scheme itself than the time stepping method.

Figure 8 shows the results for the $4 / 4$ method at a time step of $C F L=0.5$ for both the RK4 and LDDRK 4-6 time stepping methods. The improvement in dissipation and dispersion from the 4/4 scheme is evident, though the difference between the two time marching methods is small at this low time step. As the time step is increased, the LDDRK 4-6 method will show an improvement over the RK4 method.

\section{2) 1-D Shock Tube Problem}

The second one-dimensional problem solves the Category 2 shock tube problem from the First CAA Workshop. ${ }^{12}$ The equations solved are the nonlinear Euler equations, written in con-

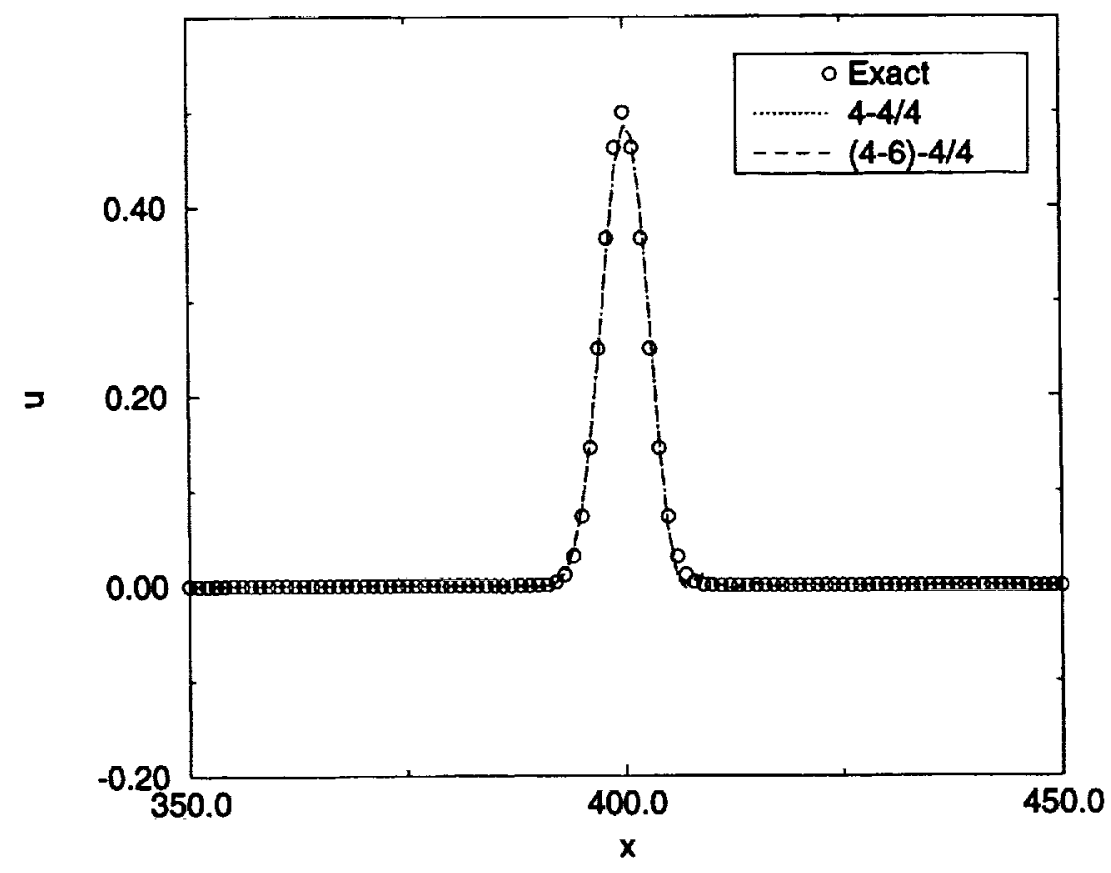

Figure 8: Performance of 4/4 Scheme on Benchmark Problem 1 
servative form:

$$
\frac{d}{d t}\left\{\begin{array}{c}
\rho \\
\rho u \\
E
\end{array}\right\}+\frac{d}{d x}\left\{\begin{array}{c}
\rho u \\
\rho u^{2}+p \\
u(E+p)
\end{array}\right\}=0
$$

where

$$
p=(\gamma-1)\left(E-\frac{1}{2} \rho u^{2}\right)
$$

The equations are solved on the domain $-235<x<235$, with $\dot{\Delta x}=1.0$. The initial conditions are:

$$
\begin{aligned}
& u(x, 0)=0 \\
& p(x, 0)=\left\{\begin{array}{cc}
4.4 & x<-2 \\
2.7+1.7 \cos \left(\frac{(x+2) \pi}{4}\right) & -2<x<2 \\
1.0 & x>2
\end{array}\right. \\
& \rho(x, 0)=(\gamma p)^{1 / \gamma}
\end{aligned}
$$

The boundary conditions used are Thompson's 1-D characteristic formulation. ${ }^{14,15}$ For 1-D, three characteristics are defined: 


$$
\begin{aligned}
& c_{1}=\frac{d p}{d t}-\bar{\rho} \bar{c} \frac{d u}{d t} \\
& c_{2}=\bar{c}^{2} \frac{d}{d t}(\rho)-\frac{d p}{d t} \\
& c_{3}=\frac{d p}{d t}+\bar{\rho} \bar{c} \frac{d u}{d t}
\end{aligned}
$$

where the overbar terms are mean values that are set as the initial values at the boundary. In this formulation, outgoing characteristics are computed using the interior scheme, while incoming characteristics are set to zero. For the inflow boundary $(x=-235), c_{1}$ and $c_{2}$ are incoming, and are set to zero. For the outflow boundary $(x=235), c_{3}$ is incoming and is set to zero.

Figures 9 and 10 show the results for density at time $=60$ for this problem. In these figures, the expansion fan is shown on the left, with the contact surface in the middle and the shock on the right. Only part of the computational domain is shown in order to illustrate the performance of the different methods.

In Fig. 9, the three time stepping methods are compared using the $4 / 2$ scheme. All three use a time step of CFL $=0.25$. The ability of all three methods to accurately track the discontinuities, with the RK2 time stepping method having more oscillations in the solution than the other two methods.

In Fig. 10, the 4/4 scheme is tested using the RK4 and LDDRK 4-6 time stepping methods. At this small time step, both methods produce comparable results. Notice that, while the $4 / 4$ method does have more oscillations in the solution compared to the $4 / 2$ method, the contact surface is more sharply resolved. This is due to the decreased dissipation and increased dispersion accuracy of the $4 / 4$ method. 


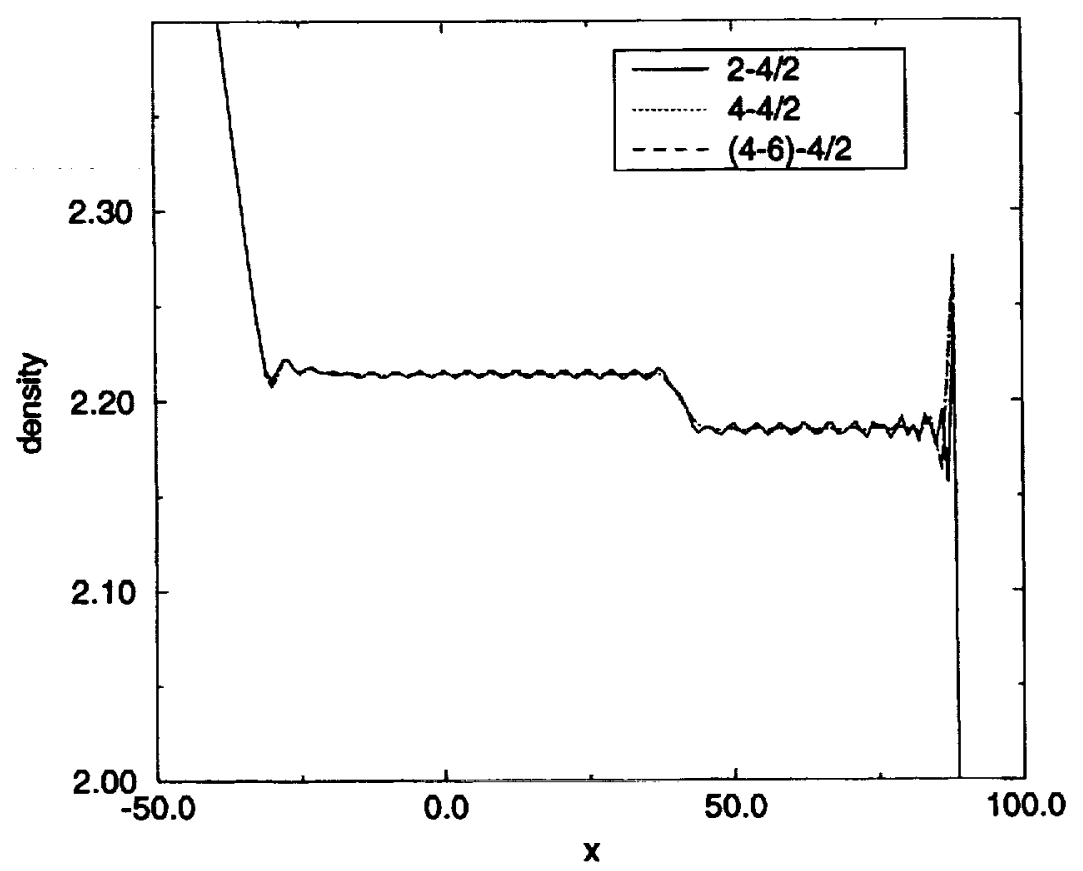

Figure 9: Effect of Time Stepping Method on 4/2 Scheme Solution of Nonlinear Benchmark Problem $2(\mathrm{CFL}=0.25)$

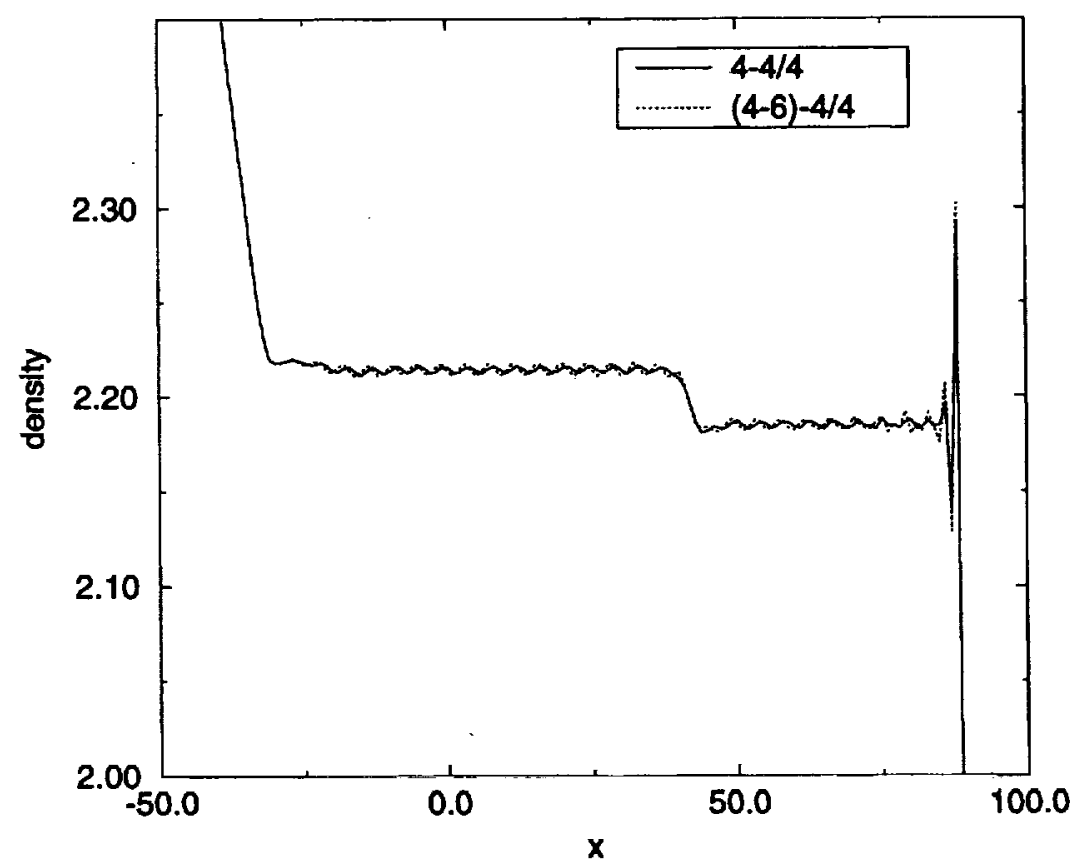

Figure 10: Effect of Time Stepping Method on 4/4 Scheme Solution of Nonlinear Benchmark Problem $2(\mathrm{CFL}=0.25)$ 


\section{3) 2-D Acoustic Scattering Problem}

The third test problem is from the Second CAA Workshop. ${ }^{13}$ In this problem, a 2-D cylinder of radius $R=0.5$ is located at the origin. At time $=0$, an initial pressure pulse is specified:

$$
p(x, y, 0)=\exp \left(-\ln (2)\left(\frac{(x-4)^{2}+y^{2}}{0.04}\right)\right)
$$

The problem asks for the unsteady pressure data for $6<\mathrm{t}<10$ at three stations: $(0,5),(5 / \sqrt{2}, 5 / \sqrt{2})$, and $(-5,0)$.

The equations to be solved are the linearized Euler equations in polar coordinates:

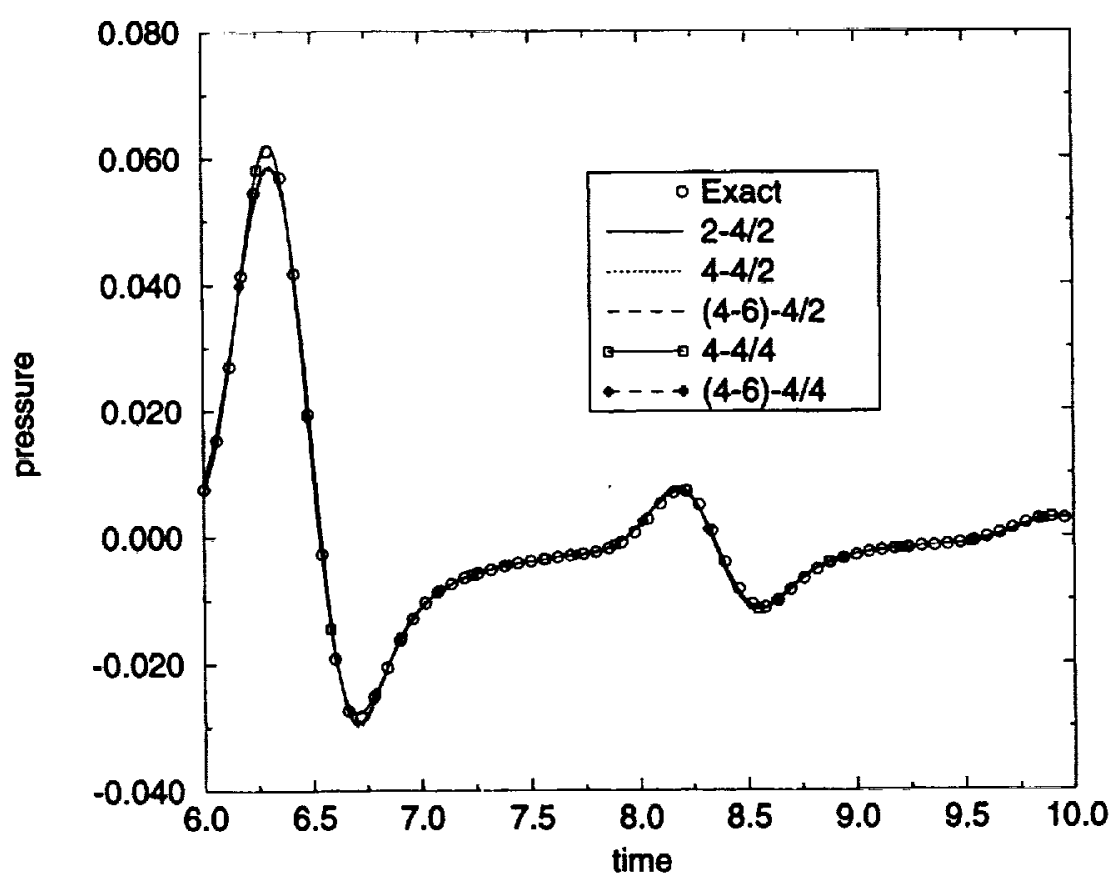

Figure 11: Solution of Benchmark Problem 3 at $(0,5)$.

$$
(\mathrm{CFL}=0.5)
$$




$$
\frac{d}{d t}\left\{\begin{array}{c}
v_{r} \\
v_{\theta} \\
p
\end{array}\right\}+\frac{d}{d r}\left\{\begin{array}{c}
p \\
0 \\
v_{r}
\end{array}\right\}+\frac{1}{r} \frac{d}{d \theta}\left\{\begin{array}{c}
0 \\
p \\
v_{\theta}
\end{array}\right\}=\frac{1}{r}\left\{\begin{array}{c}
0 \\
0 \\
v_{r}
\end{array}\right\}
$$

The computational domain extends radially from $R=0.5$ to $R=10.5$, and azimuthally from $\theta=0$ to $\theta=\pi$. Three boundary conditions are used: a wall condition on the cylinder, an acoustic radiation condition in the far field, and a periodic condition at the azimuthal boundaries.

The wall condition is based on Tam's wall condition. ${ }^{16}$ This condition requires that the time rate of change of the normal velocity at the wall is zero:

$$
\frac{d v_{r}}{d t}=\frac{d p}{d r}=0
$$

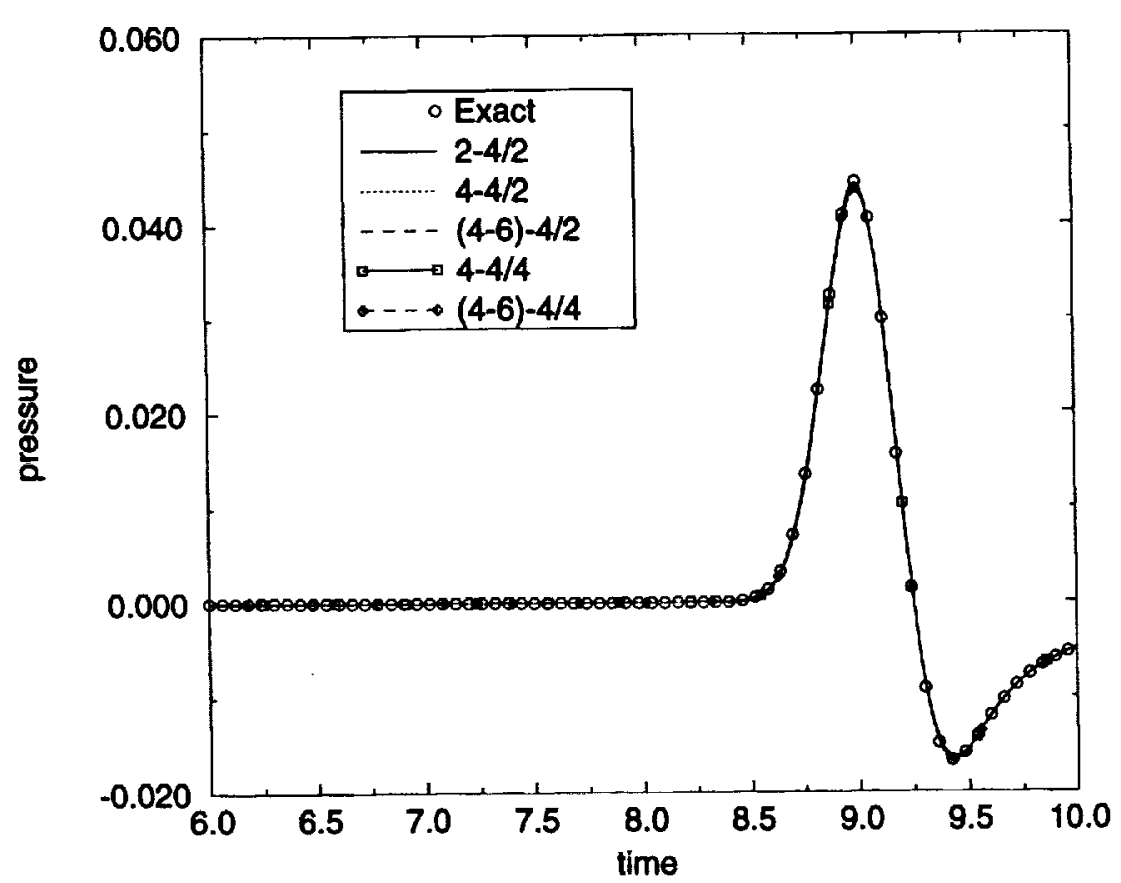

Figure 12: Solution of Benchmark Problem 3 at $(-5,0)$

$$
(\mathrm{CFL}=0.5)
$$


This condition is imposed by setting the normal derivative of pressure at the wall to zero for each sweep, while the other radial derivatives are computed normally.

The acoustic radiation boundary condition is given as:

$$
\frac{d}{d t}\left\{\begin{array}{c}
v_{r} \\
v_{\theta} \\
p
\end{array}\right\}+\frac{d}{d r}\left\{\begin{array}{c}
v_{r} \\
0 \\
p
\end{array}\right\}+\frac{1}{2 r}\left\{\begin{array}{c}
v_{r} \\
0 \\
p
\end{array}\right\}=0
$$

The radial derivatives at the exterior boundary are computed using one-sided explicit stencils. The periodic boundary condition requires some extra work. To start the sweep for the azimuthal derivative, the one-sided boundary stencil is used. At the end of the sweep, a corrected value for the derivative is known, and Eq. (34) is used to update the interior points as required.

For this calculation, a uniformly-spaced grid of 201 radial points and 301 azimuthal points is used, with a time step - CFL $=0.5$. Since the grid does not have a point at $(5 / \sqrt{2}, 5 / \sqrt{2})$, data is only taken at the points $(0,5)$ and $(-5,0)$.

Figures 11 and 12 show the results for these locations, compared to the exact solution. The results of both methods are good for this problem. At the initial transient in Fig. 11, it can be seen that the $4 / 4$ scheme is less dissipative, but has the wave arriving slightly early. The $4 / 2$ scheme is more dissipative, with a lagging dispersion error. At this relatively small time step, all time marching methods perform well. 


\section{Conclusions}

A new series of compact MacCormack-type schemes are presented which do not require tridiagonal matrix inversions. Boundary stencils and boundary conditions are described, and results are shown for linear and nonlinear CAA benchmark problems. Stability and wave propagation performance are shown for the two schemes, using various time-stepping methods. The compact methodology gives a distinct performance advantage over previous explicit schemes, and can be easily added to existing MacCormack scheme solvers.

\section{Acknowledgment}

This work was carried out under grant NCC3-531 from the NASA Lewis Research Center. Dr. L. A. Povinelli was the Technical Monitor.

\section{References}

1) Lele, S. K., 'Compact Finite Differenc Schemes with Spectral-like Resolution', Journal of Computational Physics, Vol. 103, 1992, p. 16-42.

2) Haras, Z. and Ta'asan, S., 'Finite-Difference Schemes for Long-Time Integration', J. Comp. Physics, Vol. 114, 1994, pp. 265-279.

3) Tam, C. K. W. and Webb, J. C., 'Dispersion-Relation-Preserving Finite Difference Schemes for Computational Acoustics', J. Comp. Physics, Vol. 107, 1993, p. 262-281.

4) Zingg, D. W., Lomax, H., and Jurgens, H. M., 'An Optimized Finite-Difference Scheme for Wave Propagation Problems', AIAA Paper 93-0459, Jan. 1993.

5) Gottlieb, D. and Turkel, E., 'Dissipative Two-Four Method for Time Dependent Problems', Mathematics of Computation, Vol. 30, No. 136, 1976, p.703-723.

6) Hixon, R., 'On Increasing the Accuracy of MacCormack Schemes for Aeroacoustic Applica- 
tions', AIAA Paper 97-1586, Atlanta, GA, May 1997.

7) Kreiss, H. O., Orszag, S. A., and Israeli, M., Annual Review of Fluid Mechanics, Vol 6., p. 281, 1974.

8) Hixon, R., 'A New Class of Compact Schemes', AIAA Paper 98-0367, Reno, NV, Jan. 1998.

9) Hu, F. Q., Hussaini, M. Y., and Manthey, J., 'Low-Dissipation and -Dispersion Runge-Kutta Schemes for Computational Acoustics', ICASE Report 94-102, Dec. 1994.

10) Carpenter, M. H., Gottlieb, D., and Abarbanel, S., 'Stable and Accurate Boundary Treatments for Compact, High-Order Finite-Difference Schemes', Applied Numerical Mathematics, Vol. 12, 1993, pp. 55-87

11) Carpenter, M. H., Gottlieb, D., and Abarbanel, S., 'Time-Stable Boundary Conditions for Finite-Difference Schemes Solving Hyperbolic Systems: Methodology and Application to High-Order Compact Schemes', J. Comp. Physics, Bol. 111, 1994, pp. 220-236.

12) ICASE/LARC Workshop on Benchmark Problems in Computational Aeroacoustics, ed. by J. C. Hardin, J. R. Ristorcelli, and C. K. W. Tam. NASA CP-3300, Hampton, VA, May 1995.

13) Second Computational Aeroacoustics (CAA) Workshop on Benchmark Problems, ed. by C. K.

W. Tam and J. C. Hardin. NASA CP-3352, Hampton, VA, June 1997.

14) Thompson, K. W., 'Time-Dependent Boundary Conditions for Hyperbolic Systems', J. Comp. Physics, Vol. 68, 1987, p. 1-24.

15) Thompson, K. W., 'Time-Dependent Boundary Conditions for Hyperbolic Systems, II', J. Comp. Physics, Vol. 87, 1990, p. 439-461.

16) Tam, C. K. W. and Dong, Z. 'Wall Boundary Conditions for High-Order Finite Difference Schemes in Computational Aeroacoustics', Theoret. Comput. Fluid Dynamics, Vol. 6, 


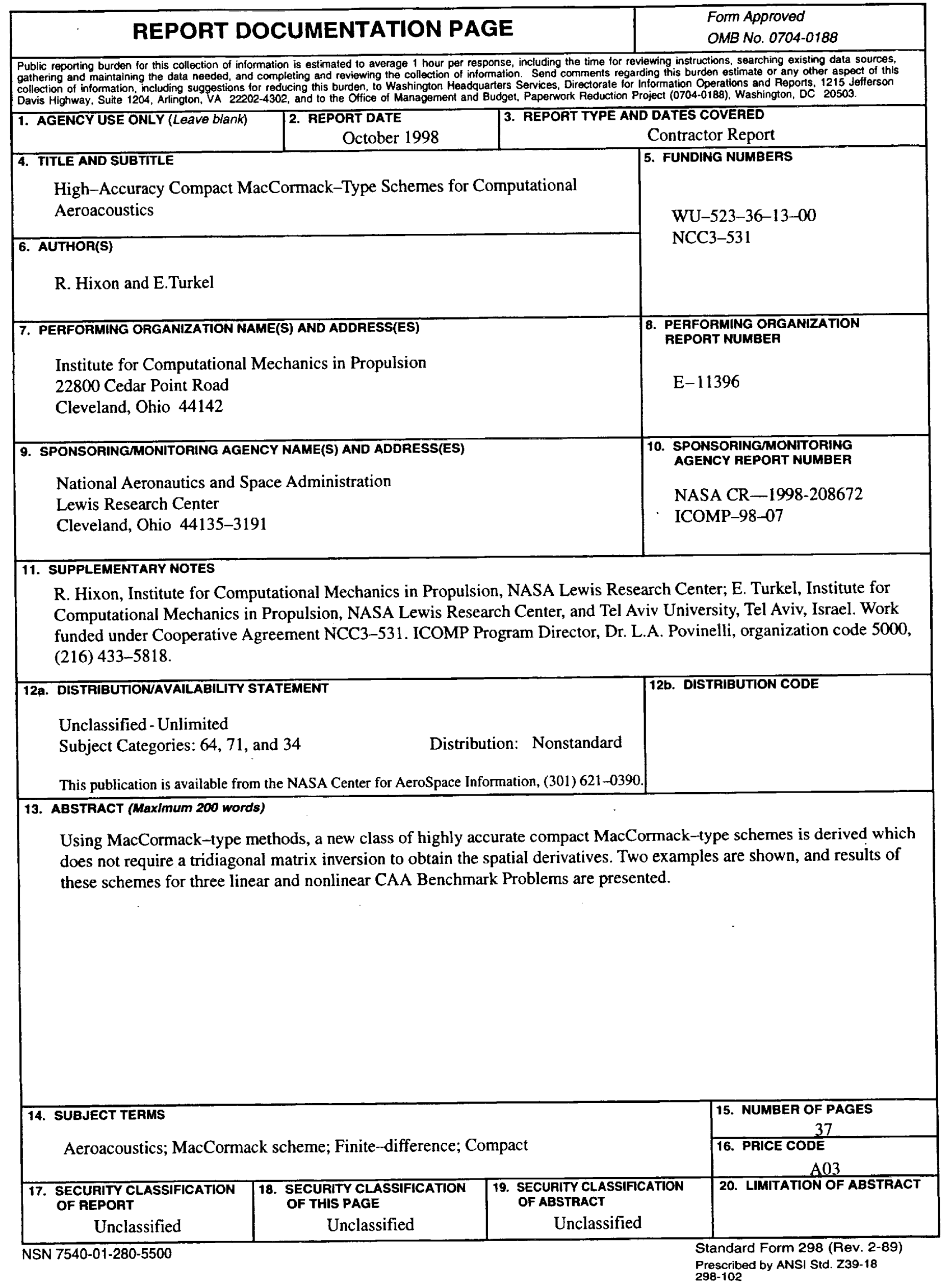


1994, p. 303-322. 